\title{
On the Associativity Functional Equation
}

\author{
Jean-Luc Marichal \\ Department of Management, FEGSS, University of Liège \\ Boulevard du Rectorat 7 - B31, B-4000 Liège, Belgium \\ jl.marichal[at]ulg.ac.be
}

Revised version, June 19, 1998

\begin{abstract}
Let $[a, b]$ be any bounded closed real interval. The class of all continuous, nondecreasing, associative functions $M:[a, b]^{2} \rightarrow[a, b]$ fulfilling the boundary conditions $M(a, a)=a$ and $M(b, b)=b$ is described.
\end{abstract}

Keywords: Associativity equation, semigroups, ordinal sums, $t$-norms and $t$-conorms.

\section{Introduction}

Let $E$ be any real interval, finite or infinite. A two-place function $M: E^{2} \rightarrow E$ is said to be associative if it fulfils the following equation

$$
M(M(x, y), z)=M(x, M(y, z)) \quad \forall x, y, z \in E .
$$

A large number of papers deal with the associativity functional equation (1), especially in the field of real numbers (for a list of references see Aczél [2, Section 6.2]). In complete generality its investigation naturally constitutes a principal subject of algebra. Many researchers tried to solve it, even under some additional conditions.

Let $[a, b]$ be a bounded closed real interval. In this paper, we intend to describe the family of continuous, nondecreasing (in each variable), associative functions $M:[a, b]^{2} \rightarrow[a, b]$ fulfilling the boundary conditions $M(a, a)=a$ and $M(b, b)=b$. This family contains all the continuous $t$-norms and $t$-conorms which are often used as aggregation tools in fuzzy sets theory, see Fodor and Roubens [8].

The paper is organized as follows. In Section 2, we simply mention the well-known description of the family of continuous, strictly increasing, associative functions. In Section 3, we recall some characterizations involving continuous, nondecreasing, associative functions. In Section 4, we present the main result as well as some corollaries. In Section 5, we investigate the case of idempotent functions. Finally, in Section 6, we examine the particular case of continuous $t$-norms and $t$-conorms.

Before entering the subject, we need to introduce some useful concepts: A semigroup $(E, M)$ is a set $E$ with an associative operation $M$ defined on it. An element $e \in E$ is

a) an identity for $M$ if $M(e, x)=M(x, e)=x$ for all $x \in E$,

b) a zero (or annihilator) for $M$ if $M(e, x)=M(x, e)=e$ for all $x \in E$,

c) an idempotent for $M$ if $M(e, e)=e$.

For any semigroup $(E, M)$, it is clear that there is at most one identity and at most one zero for $M$ in $E$, and both are idempotents.

In this paper, for any real interval $E$, we denote by $E^{\circ}$ the interior of $E$, that is the corresponding open interval. 


\section{Case of strict increasing functions}

Aczél [1] investigated the general continuous, strictly increasing (in each variable), real solution on $E^{2}$ of the associativity equation. He proved the following (see also [2, Section 6.2]).

Theorem 2.1 Let $E$ be a real interval, finite or infinite, which is open on one side. A function $M: E^{2} \rightarrow E$ is continuous, strictly increasing, and associative if and only if there exists a continuous and strictly monotonic function $f: E \rightarrow \mathbb{R}$ such that

$$
M(x, y)=f^{-1}[f(x)+f(y)] \quad \forall x, y \in E .
$$

It was also proved that the function $f$ occuring in (2) is determined up to a multiplicative constant, that is, with $f(x)$ all functions $g(x)=r f(x)\left(r \in \mathbb{R}_{0}\right)$ belongs to the same $M$, and only these.

Moreover, the function $f$ is such that, if $e \in E$ then

$$
M(e, e)=e \Leftrightarrow f(e)=0 .
$$

Indeed, if $M(e, e)=e$ then, by (2), we have $2 f(e)=f(e)$, hence $f(e)=0$. Conversely, suppose $f(e)=0$. By $(2)$, we have $0=2 f(e)=f(M(e, e))$. Since $f$ is strictly monotonic, we have $M(e, e)=e$.

By (3) and because of strict monotonicity of $f$, there is at most one idempotent for $M$ (which is, actually, the identity). Moreover, we can notice that every continuous, strictly increasing, associative function is also symmetric (i.e. $M(x, y)=M(y, x)$ for all $x, y \in E)$. The sum $(f(x)=$ $x)$ and the product $(f(x)=\log x)$ are well-known examples of continuous, strictly increasing, associative functions.

According to Ling [10], any semigroup $(E, M)$ satisfying the hypotheses of Theorem 2.1 is called Aczélian.

\section{Archimedean semigroups}

Some authors tempted to generalize Theorem 2.1 by relaxing the strict increasingness into nondecreasingness. But it seems that the class of continuous, nondecreasing, associative functions has not been described yet. However, under some additional conditions, results have been obtained.

First, we state a representation theorem attributed very often to Ling [10]. In fact, her main theorem can be deduced from previously known results on topological semigroups, see Faucett [6] and Mostert and Shields [11]. Nevertheless, the advantage of Ling's approach is twofold: treating two different cases in a unified manner and establishing elementary proofs.

Theorem 3.1 Let $E=[a, b]$. A function $M: E^{2} \rightarrow E$ is continuous, nondecreasing, associative and fulfils

$$
\begin{array}{ll}
M(b, x)=x & \forall x \in E \\
M(x, x)<x & \forall x \in E^{\circ}
\end{array}
$$

if and only if there exists a continuous strictly decreasing function $f: E \rightarrow[0,+\infty]$, with $f(b)=0$, such that

$$
M(x, y)=f^{-1}[\min (f(x)+f(y), f(a))] \quad \forall x, y \in E .
$$


The requirement that $E$ be closed is not really a restriction. If $E$ is any real interval, finite or infinite, with right endpoint $b$ ( $b$ can be $+\infty$ ), then we can replace condition (4) by

$$
\lim _{t \rightarrow b^{-}} M(t, t)=b, \quad \lim _{t \rightarrow b^{-}} M(t, x)=x \quad \forall x \in E .
$$

Any function $f$ solving equation (6) is called an additive generator (or simply generator) of $M$. Moreover, we can easily see that any function $M$ of the form (6) is symmetric and conjunctive (i.e. $M(x, y) \leq \min (x, y) \quad \forall x, y \in E)$.

Condition (4) expresses that $b$ is a left identity for $M$. It turns out, from (6), that $b$ acts as an identity, and $a$ as a zero. Condition (5) simply expresses that there are no idempotents for $M$ in $] a, b[$; indeed, by nondecreasingness and (4), we always have $M(x, x) \leq M(b, x)=x$ for all $x \in[a, b]$.

Depending on whether $f(a)$ is finite or infinite (recall that $f(a) \in[0,+\infty]$ ), $M$ takes a welldefined form (see Fodor and Roubens [8, Section 1.3] and Schweizer and Sklar [12]):

- $f(a)<+\infty$ if and only if $M$ has zero divisors (i.e. $\exists x, y \in] a, b[$ such that $M(x, y)=a$ ). In this case, there exists a continuous strictly increasing function $g:[a, b] \rightarrow[0,1]$, with $g(a)=0$ and $g(b)=1$ such that

$$
M(x, y)=g^{-1}[\max (g(x)+g(y)-1,0)] \quad \forall x, y \in[a, b] .
$$

To see this, it suffices to set $g(x):=1-f(x) / f(a)$.

- $\lim _{t \rightarrow a^{+}} f(x)=+\infty$ if and only if $M$ is strictly increasing on $] a, b[$. In this case, there exists a continuous strictly increasing function $g:[a, b] \rightarrow[0,1]$, with $g(a)=0$ and $g(b)=1$ such that

$$
M(x, y)=g^{-1}[g(x) g(y)] \quad \forall x, y \in[a, b],
$$

To see this, it suffices to set $g(x):=\exp (-f(x))$.

Of course, Theorem 3.1 can also be written under a dual form as follows.

Theorem 3.2 Let $E=[a, b]$. A function $M: E^{2} \rightarrow E$ is continuous, nondecreasing, associative and fulfils

$$
\begin{array}{ll}
M(a, x)=x & \forall x \in E \\
M(x, x)>x & \forall x \in E^{\circ}
\end{array}
$$

if and only if there exists a continuous strictly increasing function $f: E \rightarrow[0,+\infty]$, with $f(a)=0$, such that

$$
M(x, y)=f^{-1}[\min (f(x)+f(y), f(b))] \quad \forall x, y \in E .
$$

Here again, $E$ can be any real interval, even infinite. The functions $M$ of the form (11) are symmetric and disjunctive (i.e. $M(x, y) \geq \max (x, y) \quad \forall x, y \in E)$. There are no interior idempotents. The left endpoint $a$ acts as an identity and the right endpoint $b$ acts as a zero.

Once more, two mutually exclusive cases can be examined:

- $f(b)<+\infty$ if and only if $M$ has zero divisors (i.e. $\exists x, y \in] a, b[$ such that $M(x, y)=b$ ). In this case, there exists a continuous strictly increasing function $g:[a, b] \rightarrow[0,1]$, with $g(a)=0$ and $g(b)=1$ such that

$$
M(x, y)=g^{-1}[\min (g(x)+g(y), 1)] \quad \forall x, y \in[a, b] .
$$

To see this, it suffices to set $g(x):=f(x) / f(b)$. 
- $\lim _{t \rightarrow b^{-}} f(x)=+\infty$ if and only if $M$ is strictly increasing on $] a, b[$. In this case, there exists a continuous strictly increasing function $g:[a, b] \rightarrow[0,1]$, with $g(a)=0$ and $g(b)=1$ such that

$$
M(x, y)=g^{-1}[1-(1-g(x))(1-g(y))] \quad \forall x, y \in[a, b],
$$

To see this, it suffices to set $g(x):=1-\exp (-f(x))$.

Any semigroup fulfilling the assumptions of Theorem 3.1 or 3.2 is called Archimedean, see Ling [10]. In other words, when $E$ is a real interval, the semigroup $(E, M)$ is said to be Archimedean if $M$ is continuous, nondecreasing, and associative, one endpoint of $E$ is an identity for $M$, and there are no idempotents for $M$ in $E^{\circ}$. We can make a distinction between conjunctive and disjunctive Archimedean semigroups depending on whether the identity is the right endpoint of $E$ or the left endpoint of $E$, respectively. An Archimedean semigroup is called properly Archimedean or Aczélian if every additive generator $f$ is unbounded; otherwise it is improperly Archimedean.

Ling [10, Section 6] proved that every Archimedean semigroup is obtainable as a limit of Aczélian semigroups.

\section{The main result}

We now intend to describe the family $\mathcal{A}_{a, b}$ of continuous, nondecreasing, associative functions $M:[a, b]^{2} \rightarrow[a, b]$ fulfilling the boundary conditions $M(a, a)=a$ and $M(b, b)=b$. For all $\theta \in[a, b]$, we define $\mathcal{A}_{a, b, \theta}$ as the subset of functions $M \in \mathcal{A}_{a, b}$ such that $M(a, b)=M(b, a)=\theta$. The extreme cases $\mathcal{A}_{a, b, a}$ and $\mathcal{A}_{a, b, b}$ will play an important role in the sequel. From now on, $\wedge$ and $\vee$ will stand, respectively, for the minimum and maximum operations.

Theorem 4.1 $M \in \mathcal{A}_{a, b}$ if and only if there exists $\alpha, \beta \in[a, b]$ and two functions $M_{a, \alpha \wedge \beta, \alpha \wedge \beta} \in$ $\mathcal{A}_{a, \alpha \wedge \beta, \alpha \wedge \beta}$ and $M_{\alpha \vee \beta, b, \alpha \vee \beta} \in \mathcal{A}_{\alpha \vee \beta, b, \alpha \vee \beta}$ such that, for all $x, y \in[a, b]$,

$$
M(x, y)= \begin{cases}M_{a, \alpha \wedge \beta, \alpha \wedge \beta}(x, y), & \text { if } x, y \in[a, \alpha \wedge \beta] \\ M_{\alpha \vee \beta, b, \alpha \vee \beta}(x, y), & \text { if } x, y \in[\alpha \vee \beta, b] \\ (\alpha \wedge x) \vee(\beta \wedge y) \vee(x \wedge y), & \text { otherwise. }\end{cases}
$$

Proof. (Sufficiency) We can easily see that the functions $M$ defined in the statement are continuous, nondecreasing and fulfil the boundary conditions. The only property we have to prove is associativity.

Assume $\alpha \leq \beta$ (the other case can be treated similarly) and let $x, y, z \in[a, b]$. Let us show that (1) holds.

1. If $y, z \leq \alpha$ then

- if $x \leq \alpha$ then $M=M_{a, \alpha, \alpha}$ and $M(M(x, y), z)=M(x, M(y, z))$;

- if $x>\alpha$ then $M(M(x, y), z)=M(\alpha, z)=\alpha=M(x, M(y, z))$.

2. The case $y, z \geq \beta$ can be treated similarly.

3. In the remaining cases,

- if $z \leq \alpha$ and $y>\alpha$ then $M(x, y) \geq \alpha$ and $M(M(x, y), z)=\alpha=M(x, \alpha)=$ $M(x, M(y, z))$;

- if $z \geq \beta$ and $y<\beta$ then $M(x, y) \leq \beta$ and $M(M(x, y), z)=\beta=M(x, \beta)=$ $M(x, M(y, z))$;

- if $\alpha \leq z \leq \beta$ then $M(M(x, y), z)=z=M(x, z)=M(x, M(y, z))$. 
(Necessity) Set $\alpha=M(b, a), \beta=M(a, b)$ and suppose $\alpha \leq \beta$. The other case can be treated similarly.

We have

$$
\begin{aligned}
& M(\alpha, a)=M(b, \alpha)=\alpha, \\
& M(a, \beta)=M(\beta, b)=\beta .
\end{aligned}
$$

Indeed, we have for instance, $M(\alpha, a)=M(M(b, a), a)=M(b, M(a, a))=M(b, a)=\alpha$.

We have

$$
\begin{aligned}
& M(x, y)=\alpha \quad \forall x, y \in[a, b], y \leq \alpha \leq x \\
& M(x, y)=\beta \quad \forall x, y \in[a, b], x \leq \beta \leq y
\end{aligned}
$$

Indeed, we have for instance, by nondecreasingness and (14), $\alpha=M(b, \alpha) \geq M(x, y) \geq M(\alpha, a)=$ $\alpha$.

We have

$$
\begin{array}{ll}
M(a, y)=y & \forall y \in[a, \beta] \\
M(b, y)=y & \forall y \in[\alpha, b]
\end{array}
$$

Indeed, for instance, if $z$ increases from $a$ to $\beta, M(a, z)$ increases continuously from $a$ to $\beta$. Using the intermediate-value theorem, this implies that: $\forall y \in[a, \beta], \exists z \in[a, \beta]$ such that $y=M(a, z)$ and

$$
M(a, y)=M(a, M(a, z))=M(M(a, a), z)=M(a, z)=y .
$$

To end the proof, we have to prove that

$$
M(x, y)=y \quad \forall x \in[a, b] \forall y \in[\alpha, \beta] .
$$

Indeed, by nondecreasingness and (18)-(19), we simply have $y=M(a, y) \leq M(x, y) \leq M(b, y)=$ $y$.

As we can note, the previous characterization partitions the definition set $[a, b]^{2}$ into several pieces. On each one of them, $M$ takes a well defined form. Figure 1 presents graphics showing this partition and the corresponding values of the function.

Now, our task consists in describing the two families $\mathcal{A}_{a, b, a}$ and $\mathcal{A}_{a, b, b}$. For this purpose, consider a proposition.

Proposition 4.1 For any continuous, nondecreasing, associative functions $M:[a, b]^{2} \rightarrow$ $[a, b]$, the following assertions are equivalent:
i) $b$ is an identity for $M$
ii) $a$ is a zero, and $b$ is an idempotent for $M$
iii) $M(a, b)=M(b, a)=a$, and $b$ is an idempotent for $M$

The assertions remain equivalent if the endpoints $a$ and $b$ are exchanged.

Proof. $i)$ or $i i) \Rightarrow$ iii) Trivial.

iii $\Rightarrow$ ii) For all $x \in[a, b]$, we have $M(a, x) \leq M(a, b)=a$, so that $M(a, x)=a$.

$i i i) \Rightarrow i$ ) If $z$ increases from $a$ to $b, M(b, z)$ increases continuously from $a$ to $b$. Using the intermediate-value theorem, this implies that: $\forall x \in[a, b], \exists z \in[a, b]$ such that $x=M(b, z)$ and

$$
M(b, x)=M(b, M(b, z))=M(M(b, b), z)=M(b, z)=x .
$$

We can prove similarly that $M(x, b)=x$ for all $x \in[a, b]$.

Before going on, we need to introduce the concept of ordinal sum, well-known in the theory of semigroups (see e.g. [10]). 


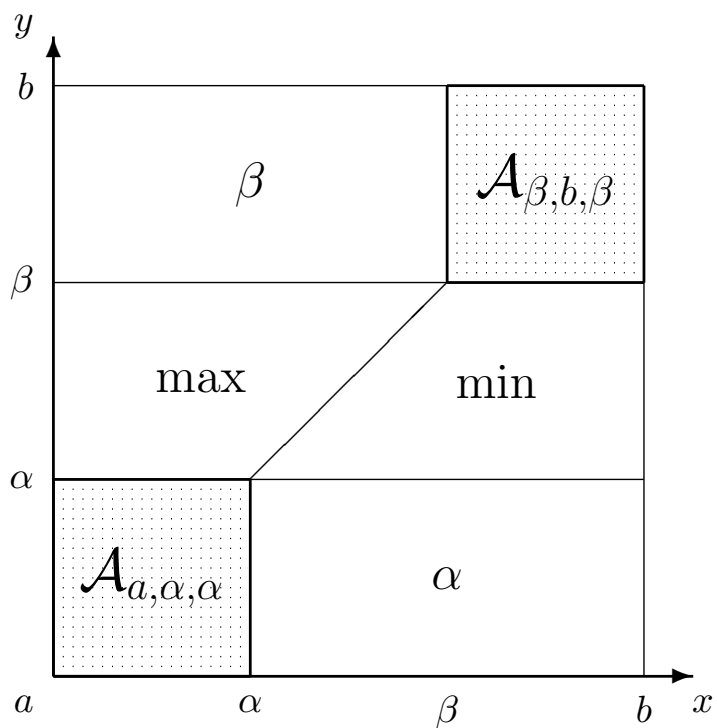

Case of $\alpha \leq \beta$

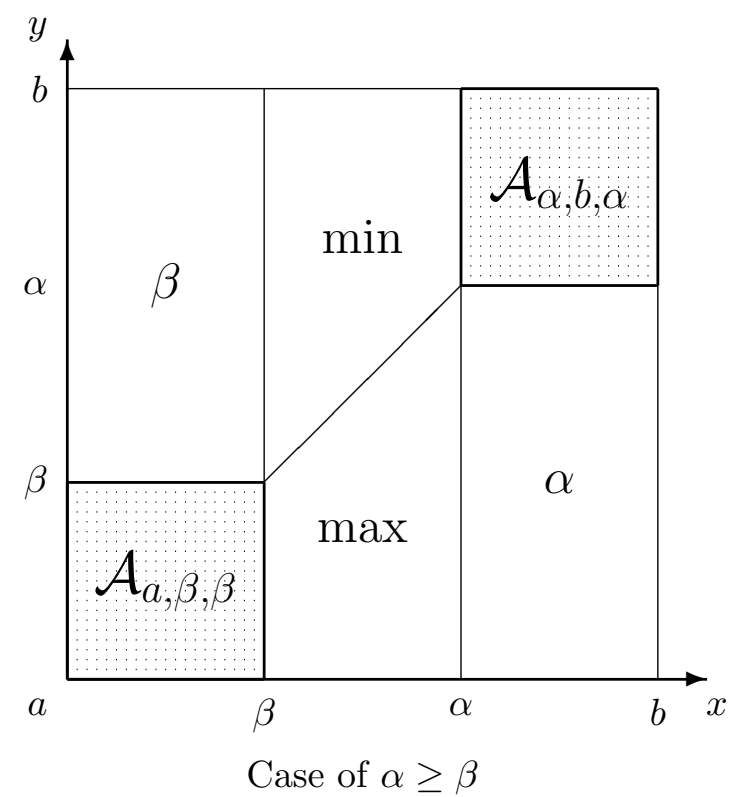

Case of $\alpha \geq \beta$

Figure 1: Description of $\mathcal{A}_{a, b}$

Definition 4.1 Let $K$ be a totally ordered set and $\left\{\left(E_{k}, M_{k}\right) \mid k \in K\right\}$ be a collection of disjoint semigroups indexed by $K$. Then the ordinal sum of $\left\{\left(E_{k}, M_{k}\right) \mid k \in K\right\}$ is the set-theoretic union $\cup_{k \in K} E_{k}$ under the following binary operation:

$$
M(x, y)= \begin{cases}M_{k}(x, y), & \text { if } \exists k \in K \text { such that } x, y \in E_{k} \\ \min (x, y), & \text { if } \exists k_{1}, k_{2} \in K, k_{1} \neq k_{2} \text { such that } x \in E_{k_{1}} \text { and } y \in E_{k_{2}} .\end{cases}
$$

The ordinal sum is a semigroup under the above defined operation.

Now, let us turn to the description of $\mathcal{A}_{a, b, a}$. Mostert and Shields [11, p. 130, Theorem B] proved the following.

Theorem 4.2 A function $M:[a, b]^{2} \rightarrow[a, b]$ is continuous, associative, and is such that a acts as a zero and $b$ as an identity if and only if

- either

$$
M(x, y)=\min (x, y) \quad \forall x, y \in[a, b]
$$

- or there exists a continuous strictly decreasing function $f:[a, b] \rightarrow[0,+\infty]$, with $f(b)=0$, such that

$$
M(x, y)=f^{-1}[\min (f(x)+f(y), f(a))] \quad \forall x, y \in[a, b] .
$$

(conjunctive Archimedean semigroup)

- or there exist a countable index set $K \subseteq \mathbb{N}$, a family of disjoint open subintervals \{]$a_{k}, b_{k}[\mid k \in$ $K\}$ of $[a, b]$ and a family $\left\{f_{k} \mid k \in K\right\}$ of continuous strictly decreasing function $f_{k}:\left[a_{k}, b_{k}\right] \rightarrow$ $[0,+\infty]$, with $f_{k}\left(b_{k}\right)=0$, such that, for all $x, y \in[a, b]$,

$$
M(x, y)= \begin{cases}f_{k}^{-1}\left[\min \left(f_{k}(x)+f_{k}(y), f_{k}\left(a_{k}\right)\right)\right], & \text { if } \exists k \in K \text { such that } x, y \in\left[a_{k}, b_{k}\right] \\ \min (x, y), & \text { otherwise. }\end{cases}
$$

(ordinal sum of conjunctive Archimedean semigroups and one-point semigroups) 


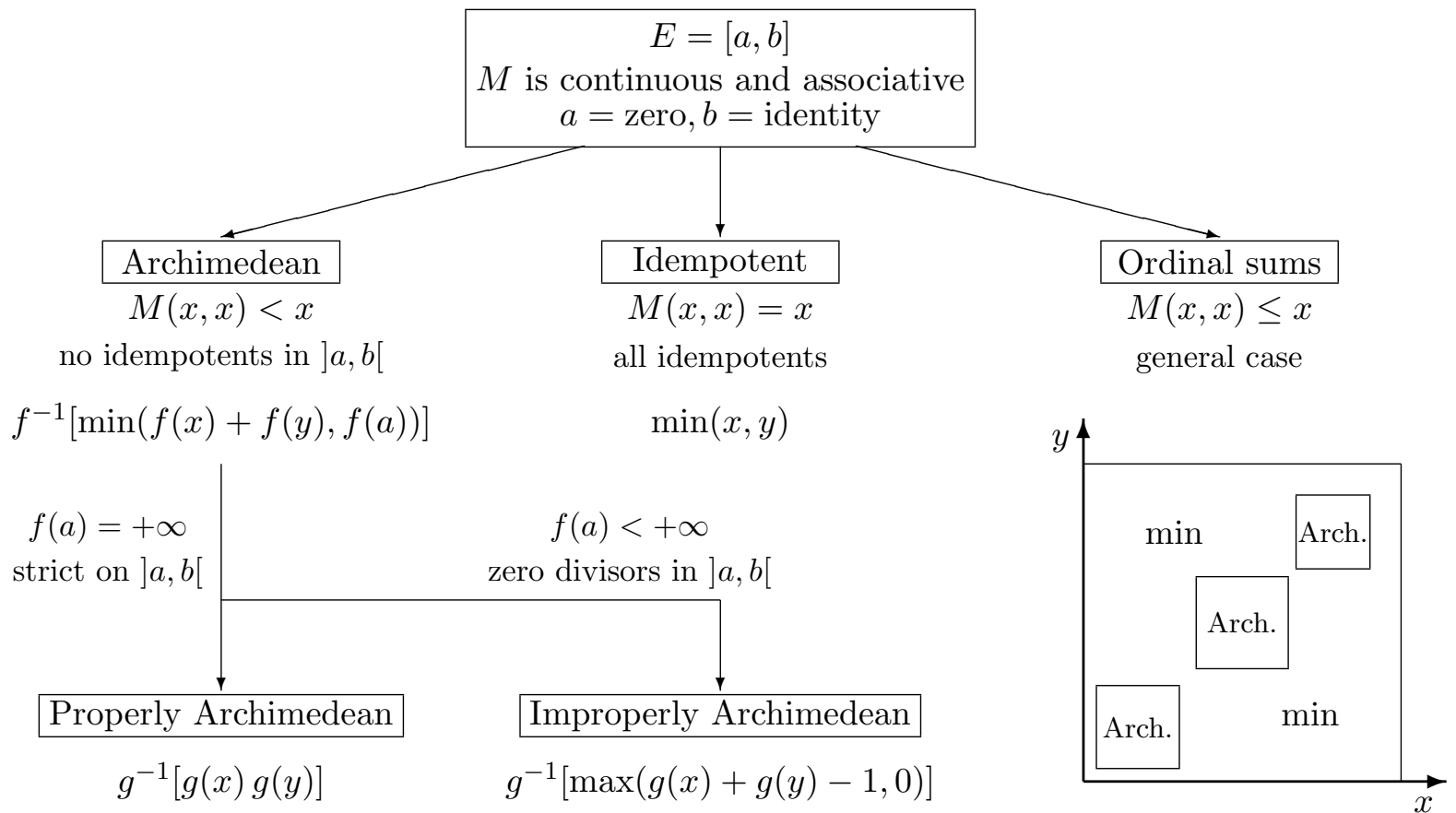

Figure 2: Description of $\mathcal{A}_{a, b, a}$

By Proposition 4.1, $\mathcal{A}_{a, b, a}$ is the family of continuous, nondecreasing, associative functions $M:[a, b]^{2} \rightarrow[a, b]$ such that $a$ acts as a zero and $b$ as an identity. Consequently, the description of the family $\mathcal{A}_{a, b, a}$ is also given by Theorem 4.2 (see also Figure 2). Moreover, it turns out that all functions fulfilling the assumptions of this result are also symmetric, nondecreasing and conjunctive.

Theorem 4.2 can also be written under a dual form as follows.

Theorem 4.3 A function $M:[a, b]^{2} \rightarrow[a, b]$ is continuous, associative, and is such that a acts as an identity and $b$ as a zero if and only if

- either

$$
M(x, y)=\max (x, y) \quad \forall x, y \in[a, b]
$$

- or there exists a continuous strictly increasing function $f:[a, b] \rightarrow[0,+\infty]$, with $f(a)=0$, such that

$$
M(x, y)=f^{-1}[\min (f(x)+f(y), f(b))] \quad \forall x, y \in[a, b] .
$$

(disjunctive Archimedean semigroup)

- or there exist a countable index set $K \subseteq \mathbb{N}$, a family of disjoint open subintervals \{]$a_{k}, b_{k}[\mid k \in$ $K\}$ of $[a, b]$ and a family $\left\{f_{k} \mid k \in K\right\}$ of continuous strictly increasing function $f_{k}:\left[a_{k}, b_{k}\right] \rightarrow$ $[0,+\infty]$, with $f_{k}\left(a_{k}\right)=0$, such that, for all $x, y \in[a, b]$,

$$
M(x, y)= \begin{cases}f_{k}^{-1}\left[\min \left(f_{k}(x)+f_{k}(y), f_{k}\left(b_{k}\right)\right)\right], & \text { if } \exists k \in K \text { such that } x, y \in\left[a_{k}, b_{k}\right] \\ \max (x, y), & \text { otherwise. }\end{cases}
$$

(ordinal sum of disjunctive Archimedean semigroups and one-point semigroups)

As above, we can see that $\mathcal{A}_{a, b, b}$ is the family of continuous, nondecreasing, associative functions $M:[a, b]^{2} \rightarrow[a, b]$ such that $a$ acts as an identity and $b$ as a zero. The description of the family 


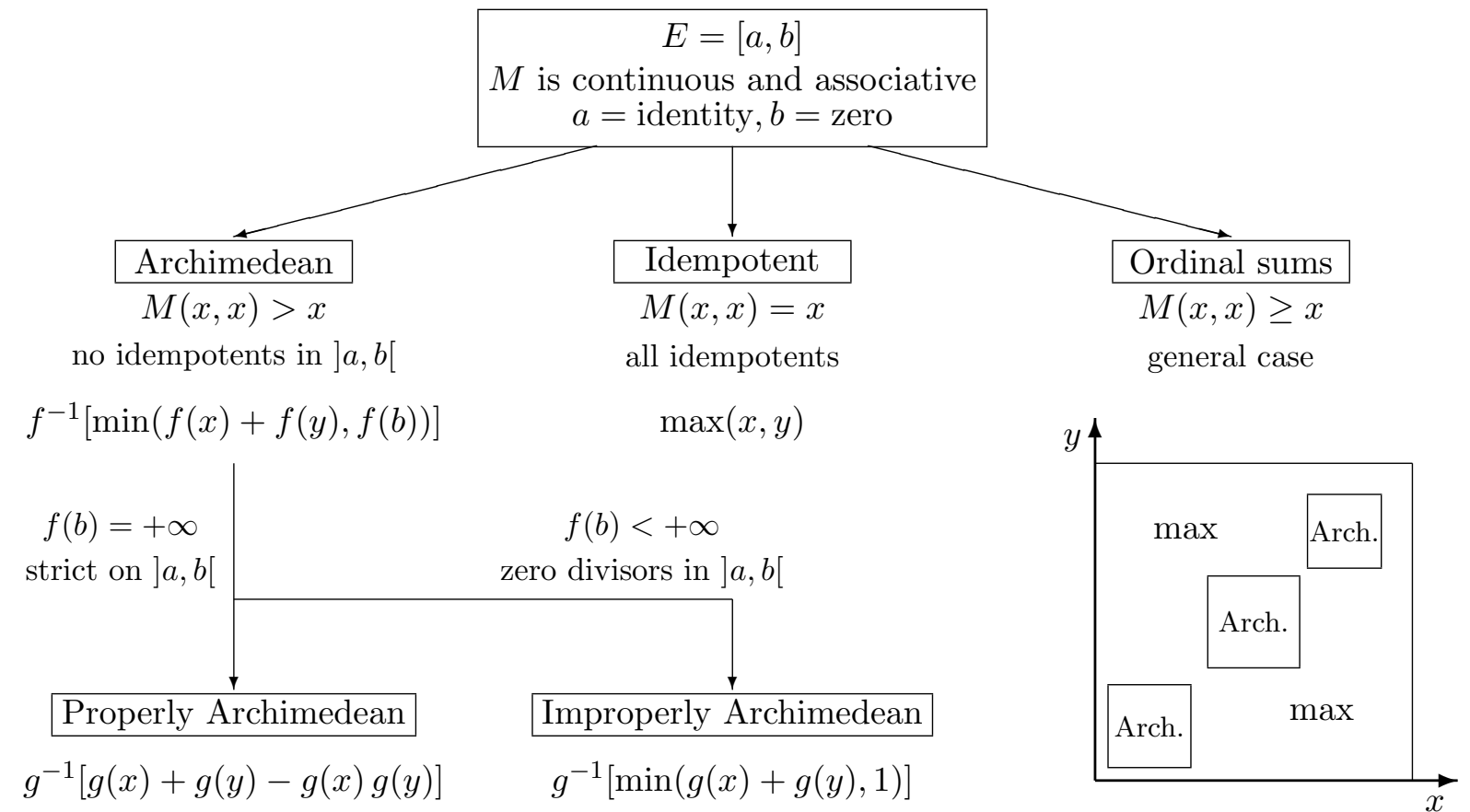

Figure 3: Description of $\mathcal{A}_{a, b, b}$

$\mathcal{A}_{a, b, b}$ is thus given by Theorem 4.3 (see also Figure 3). Moreover, all functions fulfilling the assumptions of this result are also symmetric, nondecreasing and disjunctive.

Theorems 4.1, 4.2 and 4.3, taken together, give a complete description of the family $\mathcal{A}_{a, b}$. Imposing some additional conditions leads to the following immediate corollaries.

Corollary 4.1 $M \in \mathcal{A}_{a, b}$ and is strictly increasing (in each variable) if and only if there exists a continuous strictly increasing function $g:[a, b] \rightarrow[0,1]$, with $g(a)=0$ and $g(b)=1$ such that

- either

$$
M(x, y)=g^{-1}[g(x) g(y)] \quad \forall x, y \in[a, b],
$$

- or

$$
M(x, y)=g^{-1}[g(x)+g(y)-g(x) g(y)] \quad \forall x, y \in[a, b] .
$$

Corollary 4.2 $M \in \mathcal{A}_{a, b}$ and is symmetric if and only if there exist $\alpha \in[a, b]$ and two functions $M_{a, \alpha, \alpha} \in \mathcal{A}_{a, \alpha, \alpha}$ and $M_{\alpha, b, \alpha} \in \mathcal{A}_{\alpha, b, \alpha}$ such that, for all $x, y \in[a, b]$,

$$
M(x, y)= \begin{cases}M_{a, \alpha, \alpha}(x, y), & \text { if } x, y \in[a, \alpha] \\ M_{\alpha, b, \alpha}(x, y), & \text { if } x, y \in[\alpha, b] \\ \alpha, & \text { otherwise }\end{cases}
$$

Corollary 4.3 $M \in \mathcal{A}_{a, b}$ and has exactly one identity element in $[a, b]$ if and only if $M \in$ $\mathcal{A}_{a, b, a} \cup \mathcal{A}_{a, b, b}$.

\section{Idempotent functions}

Now, we investigate the case of idempotent functions (i.e. $M(x, x)=x$ for all $x \in[a, b]$ ). Although we have seen in Section 2 that there is no continuous, strictly increasing, associative functions 

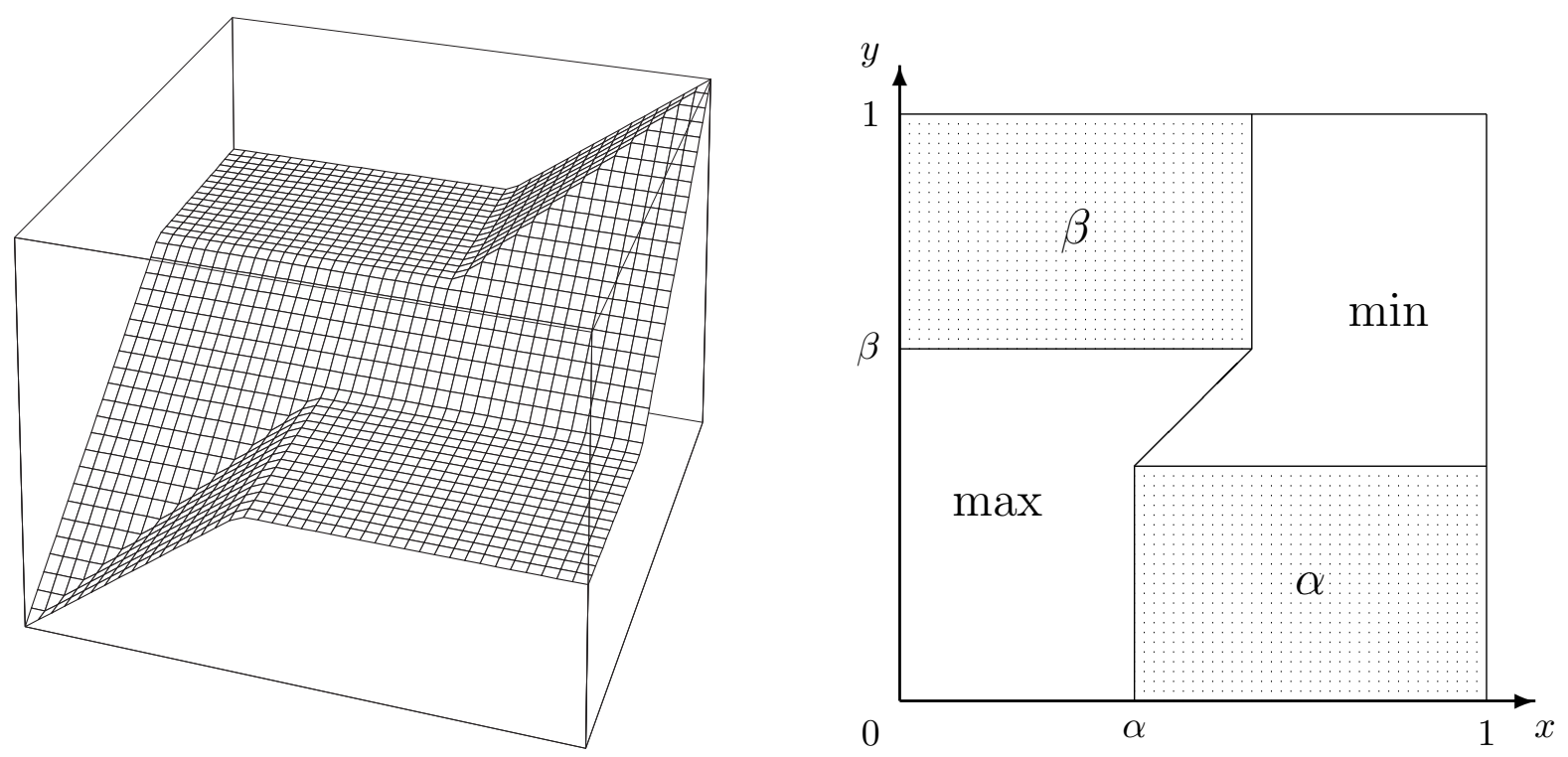

Figure 4: Representation on $[0,1]^{2}$ of $M(x, y)=(\alpha \wedge x) \vee(\beta \wedge y) \vee(x \wedge y)$ in case of $\alpha \leq \beta$

which are idempotent, the class of continuous, nondecreasing, idempotent, associative functions is not empty and its description can be deduced from Theorem 4.1. However, Fodor [7] had already obtained this description in a more general framework. In his result, $E$ can be any connected order topological space. In particular, $E$ can be an arbitrary real interval, even infinite.

Theorem 5.1 Let $E$ be a real interval, finite or infinite. A function $M: E^{2} \rightarrow E$ is continuous, nondecreasing, idempotent, and associative if and only if there exist $\alpha, \beta \in E$ such that

$$
M(x, y)=(\alpha \wedge x) \vee(\beta \wedge y) \vee(x \wedge y) \quad \forall x, y \in E .
$$

Notice that, by distributivity of $\wedge$ and $\vee, M$ can be written also in the equivalent form:

$$
M(x, y)=(\beta \vee x) \wedge(\alpha \vee y) \wedge(x \vee y) \quad \forall x, y \in E .
$$

On the basis of (20), the graphical representation of $M$ can be drawn, see Figure 4 .

Before Fodor [7], the symmetric case was obtained by Fung and $\mathrm{Fu}[9]$ and in a revisited way by Dubois and Prade [5]. Now, the result can be formulated as follows.

Theorem 5.2 Let $E$ be a real interval, finite or infinite. A function $M: E^{2} \rightarrow E$ is symmetric, continuous, nondecreasing, idempotent, and associative if and only if there exists $\alpha \in E$ such that

$$
M(x, y)=\operatorname{median}(x, y, \alpha) \quad \forall x, y \in E
$$

Czogala and Drewniak [4] have examined the case when $M$ has an identity element $e \in E$. They obtained the following.

Theorem 5.3 Let $E$ be a real interval, finite or infinite.

i) If $M: E^{2} \rightarrow E$ is nondecreasing, idempotent, associative, and has an identity element $e \in E$, then there is a nonincreasing function $g: E \rightarrow E$ with $g(e)=e$ such that, for all $x, y \in E$,

$$
M(x, y)= \begin{cases}x \wedge y, & \text { if } y<g(x) \\ x \vee y, & \text { if } y>g(x) \\ x \wedge y \text { or } x \vee y, & \text { if } y=g(x) .\end{cases}
$$


ii) If $M: E^{2} \rightarrow E$ is continuous, nondecreasing, idempotent, associative, and has an identity element $e \in E$, then $M=\min$ or $\max$.

Fodor [7] showed that the previous result still holds in the more general framework of connected order topological spaces.

\section{Triangular norms and conorms}

In fuzzy set theory, one of the main topics consists in defining fuzzy logical connectives which are appropriate extensions of logical connectives AND, OR and NOT in the case when the valuation set is the unit interval $[0,1]$ rather than $\{0,1\}$ (see e.g. [8]).

Fuzzy connectives modelling AND and OR are called triangular norms ( $t$-norms for short) and triangular conorms (t-conorms) respectively, see [3, 12].

Definition 6.1 i) $A$ t-norm is a symmetric, nondecreasing, associative function $T:[0,1]^{2} \rightarrow$ $[0,1]$ having 1 as identity.

ii) A t-conorm is a symmetric, nondecreasing, associative function $S:[0,1]^{2} \rightarrow[0,1]$ having 0 as identity.

The family of continuous $t$-norms is nothing less than the class $\mathcal{A}_{0,1,0}$, and the family of continuous $t$-conorms is the class $\mathcal{A}_{0,1,1}$. These families have been described in Section 4 . Moreover, in this context, Corollary 4.3 gives a characterization of their union.

Corollary 6.1 $M \in \mathcal{A}_{0,1}$ and has exactly one identity element in $[0,1]$ if and only if $M$ is a continuous t-norm or a continuous t-conorm.

We will not stress on this topic of $t$-norms and $t$-conorms. For a good survey and references, see Fodor and Roubens [8].

\section{Acknowledgement}

The author gratefully acknowledges partial support by NATO (grant CRG 931531).

\section{References}

[1] J. Aczél, Sur les opérations définies pour des nombres réels, Bull. Soc. Math. de France 76 (1948) 59-64.

[2] J. Aczél, Lectures on Functional Equations and Applications, (Academic Press, New York, 1966).

[3] C. Alsina, E. Trillas and L. Valverde, On some logical connectives for fuzzy sets theory, J. Math. Anal. Appl. 93 (1) (1983) 15-26.

[4] E. Czogała and J. Drewniak, Associative monotonic operations in fuzzy set theory, Fuzzy Sets and Systems 12 (1984) 249-269.

[5] D. Dubois and H. Prade, Criteria aggregation and ranking of alternatives in the framework of fuzzy set theory, in: H.-J. Zimmermann, L.A. Zadeh and B. Gaines (Eds.), Fuzzy Sets and Decision Making, TIMS/Studies in Management Sciences 20 (1984) 209-240. 
[6] W.M. Faucett, Compact semigroups irreducibly connected between two idempotents, Proc. Amer. Math. Soc. 6 (1955) 741-747.

[7] J.C. Fodor, An extension of Fung-Fu's theorem, International Journal of Uncertainty, Fuzziness and Knowledge-Based Systems, Vol. 4, No. 3 (1996) 235-243.

[8] J.C. Fodor and M. Roubens, Fuzzy Preference Modelling and Multicriteria Decision Support, (Kluwer, Dordrecht, 1994).

[9] L.W. Fung and K.S. Fu, An axiomatic approach to rational decision making in a fuzzy environment, in: Fuzzy Sets and their Applications to Cognitive and Decision Processes, eds. L.A. Zadeh, K.S. Fu, K. Tanaka and M. Shimura, (Academic Press, New York-San FranciscoLondon, 1975) 227-256.

[10] C.H. Ling, Representation of associative functions, Publ. Math. Debrecen, 12 (1965) 189-212.

[11] P.S. Mostert and A.L. Shields, On the structure of semigroups on a compact manifold with boundary, Ann. of Math. 65 (1957) 117-143.

[12] B. Schweizer and A. Sklar, Probabilistic Metric Spaces, (North-Holland, Amsterdam, 1983). 